\title{
Surveillance for pneumococcal disease in the United States
}

\author{
Cynthia G Whitney MD MPH
}

\section{WHY CONDUCT SURVEILLANCE FOR PNEUMOCOCCAL DISEASE?}

Worldwide, infections with Streptococcus pneumoniae are among the leading causes of illness and death among infants and young children, persons with chronic medical conditions, and the elderly. In the United States, pneumococcal infections cause an estimated 3000 episodes of meningitis, 50,000 episodes of sepsis, 120,000 episodes of pneumonia, and as many as seven million cases of otitis media each year (1). The mortality rate for invasive pneumococcal infections (infections of blood or other sterile sites) is high (15\% to $20 \%$ ) among adults; among the elderly, mortality can reach $40 \%$.

Despite its public health importance, until recently few public health agencies have included pneumococcal disease as a part of routine disease surveillance. However, with the emergence of drug-resistant Streptococcus pneumoniae (DRSP), the need for information about disease trends has increased. In some settings, local surveillance data, which include reporting of antimicrobial susceptibility results, can help clinicians with treatment decisions; at a higher level, regional or national trend information can guide those drafting treatment guidelines. As treatment of pneumococcal infections becomes more difficult, renewed efforts should focus on preventing disease with pneumococcal vaccines; public health practitioners can use surveillance data to target vaccination programs and to measure program impact.

This article examines the issues surrounding surveillance for pneumococcal disease and describes surveillance systems in use in the United States. In addition, the article reviews the epidemiology of pneumococcal disease and the emergence of drug-resistant infections, emphasizing surveillance findings.

\section{PLANNING SURVEILLANCE FOR DRSP}

Several issues must be considered when planning a surveillance system for pneumococcal disease and when interpreting surveillance data. The most important factor to consider may be an assessment of the laboratory methods used by reporting institutions to measure antimicrobial susceptibility. In 1994, the Centers for Disease Control and Prevention (CDC), Atlanta, Georgia convened a working group of public health practitioners, clinical laboratory personnel, health-care providers and representatives of key professional societies to develop a strategy to address DRSP; one objective of this group was to promote the use of appropriate methods for identification of DRSP (2). The working group recommended that all pneumococcal isolates from normally sterile sites be tested for resistance to penicillin according to guidelines from the National Committee for Clinical Laboratory Standards (NCCLS), Villanova, Pennsylvania (3). Isolates can be screened initially for penicillin resistance using a $1 \mu \mathrm{g}$ oxacillin disk; isolates possibly resistant to penicillin (oxacillin zone size $19 \mathrm{~mm}$ or less) should undergo quantitative minimum inhibitory concentration (MIC) testing. Recommended methods for MIC testing include broth microdilution, antimicrobial gradient strips, disk diffusion and agar dilution. Because automated systems for measuring MICs may not provide a valid and reliable measurement for pneumococci, the NCCLS does not recommend their use (4).

Respiratory Diseases Branch, Division of Bacterial and Mycotic Diseases, National Center for Infectious Diseases, Centers for Disease Control and Prevention, Atlanta, Georgia

Correspondence: Dr CG Whitney, CDC Mailstop C-23, 1600 Clifton Road Northeast, Atlanta, Georgia 30333. Telephone 404-639-4727, fax404-639-3970,e-mail cgw3@cdc.gov 
Another concern for surveillance of DRSP is the use of interpretive standards in susceptibility testing. Based on the MIC values, the NCCLS provides definitions of susceptible, intermediate and resistant isolates for several antimicrobial agents. For pneumococci, an MIC of $0.06 \mu \mathrm{g} / \mathrm{mL}$ or less is considered susceptible to penicillin, an MIC of 0.12 to $1.0 \mu \mathrm{g} / \mathrm{mL}$ is considered intermediate, and an MIC of $2.0 \mu \mathrm{g} / \mathrm{mL}$ or greater is considered resistant (4). The definitions for some drugs have changed in recent years; surveillance for DRSP should allow reporting of MIC values or account for any differences that may exist between definitions of susceptible, intermediate and resistant used by reporting laboratories and those currently recommended by NCCLS.

The population selected for surveillance can affect interpretation of results. A population-based surveillance system in nine sites in the United States and Canada found wide variation among the sites, in both the incidence of disease and the proportion of isolates that were drug-resistant (5). The proportion of penicillin-resistant strains among the sites ranged from $1.7 \%$ (Toronto, Ontario) to $19.3 \%$ (Tennessee). Among hospitals within a single site, the proportion of resistant isolates also varied markedly. Within Georgia, for example, the proportion of penicillin-resistant isolates ranged from $4.6 \%$ to $30.6 \%$; in Tennessee, the proportion ranged from $8.5 \%$ to $38.2 \%$. The choice of reportable conditions also can affect results because the proportion of drug-resistant isolates may differ for invasive and noninvasive pneumococcal infections.

\section{SURVEILLANCE SYSTEMS IN THE UNITED STATES}

Since 1979, CDC has conducted surveillance for invasive pneumococcal disease. The first system was a voluntary sentinel surveillance system designed to assess serotype distribution and antimicrobial susceptibility. Over time, the system has involved 12 to 54 hospitals in 11 to 26 states. With this system, researchers at participating hospitals collect all pneumococcal isolates from sterile sites along with some demographic and clinical information. The isolates are sent to the CDC for serotyping and susceptibility testing.

In 1994, CDC began active, population-based surveillance for invasive pneumococcal disease. This program was added to an existing active surveillance system, which was established in 1989 to monitor other infectious diseases. Active surveillance for invasive pneumococcal infections is ongoing among a population of 18.5 million persons in parts of eight states (California, Connecticut, Georgia, Maryland, Minnesota, New York, Oregon and Tennessee).

Data collection through the active surveillance system is labour intensive. To detect patients with $S$ pneumoniae isolated from blood or other normally sterile body sites, surveillance personnel have regular contact with all microbiology laboratory directors or hospital infection control practitioners in their areas. Clinical isolates are collected and tested for antimicrobial susceptibility at two reference laboratories. Surveillance personnel also complete a standardized report form for each case. Information collected for the report form includes basic demographic data and underlying medical condi- tions of the patient and is gathered by reviewing medical records or through contact with physicians or infection control practitioners.

In 1994, the Council of State and Territorial Epidemiologists resolved that each state should designate as reportable all invasive infections caused by drug-resistant pneumococci. Since then, a number of states have mandated reporting of DRSP. A survey conducted by CDC in 1996 found that 16 (31\%) of 52 state and territorial health jurisdictions had designated DRSP reportable and that $12(23 \%)$ jurisdictions were planning to do so by June 1997 (6). Of the 28 jurisdictions that were conducting surveillance or planning to initiate surveillance, seven $(25 \%)$ were collecting or planning to collect information on all invasive pneumococcal infections (including those caused by susceptible isolates) to enable estimation of the proportion not susceptible to antimicrobial agents; $19(68 \%)$ were collecting or planning to collect information on infections caused by intermediate and resistant isolates only. Surveillance in seven jurisdictions included reporting of drug-resistant pneumococcal infections with noninvasive as well as invasive isolates.

A number of other surveillance programs monitor drugresistant pneumococcal infections in addition to those operated by state and federal agencies. These other programs include groups of academic medical institutions that have collaborated to study clinical issues related to drug-resistant infections. In addition, private corporations have started isolate collections primarily for research and development of new antimicrobial agents. These surveillance systems use isolates from a variety of sources (invasive and/or noninvasive) and populations.

\section{SURVEILLANCE RESULTS: EPIDEMIOLOGY OF INVASIVE PNEUMOCOCCAL DISEASE}

Findings from population-based studies indicate that the incidence of all invasive pneumococcal infections in the United States is between 15 and 30 infections per 100,000 population. Incidence rates are higher for persons 65 years of age and older (50 to 83 cases per 100,000 population) and for children younger than age two years (approximately 160 cases per 100,000 population) (1). The incidence of invasive infections varies among communities and among racial and ethnic groups within the United States; the incidence rate of invasive pneumococcal disease for African-Americans and for certain American Indian groups is three to five times higher than the rate for Caucasians. Of 90 known pneumococcal serotypes, an estimated $85 \%$ of invasive disease among persons older than age 65 years of age is caused by one of the serotypes contained in the currently available 23-valent pneumococcal polysaccharide vaccine (7).

The type of pneumococcal infection varies by age. In adults, most pneumococcal bacteremia is associated with pneumonia; in children, the source for bacteremic infections is usually not identified (1). Pneumococcal meningitis is most common among children younger than two years of age. Since the decline of Haemophilus influenzae type b disease following widespread vaccination, pneumococcal meningitis is now 
the most common type of meningitis in the United States (incidence 1.1 case per 100,000 population) (8).

During the early and mid-1980s, drug resistance had already become a problem in several countries. In the United States during that period, very few pneumococcal isolates were resistant to penicillin (MIC $2 \mu \mathrm{g} / \mathrm{mL}$ or greater), and only $5 \%$ were intermediate to penicillin (MIC 0.1 to $1 \mu \mathrm{g} / \mathrm{mL}$ ), according to the susceptibility results of isolates submitted to CDC's Sentinel Surveillance System (9). However, by 1991 and $1992,1.3 \%$ of isolates submitted to the Sentinel Surveillance System were resistant to penicillin, $5.4 \%$ were intermediate, and $0.6 \%$ were resistant to cefotaxime (10). The trend continued during 1993 and 1994 , with $14.1 \%$ of isolates intermediate and $3.2 \%$ resistant to penicillin (7). The emergence of beta-lactam resistance during this period was accompanied by an increase in the resistance to other drugs and an increase in the proportion of isolates resistant to two and three classes of antimicrobial agents.

CDC's Active Surveillance System provides more recent data about DRSP. From July 1995 to June 1996, 10.9\% (457 of 4192 sterile site isolates) and $10.1 \%$ of pneumococcal isolates were intermediate and resistant to penicillin, respectively, and $5.4 \%$ and $4.6 \%$ were intermediate and resistant to cefotaxime (11). Among other agents tested, approximately $10 \%$ were not susceptible (intermediate or resistant) to erythromycin, and $25 \%$ were not susceptible to trimethoprim/sulfamethoxazole. Notably, $19.8 \%$ and $9.4 \%$ of isolates were not susceptible to two and three classes of antimicrobial agents, respectively. No isolates have been identified that are not susceptible to vancomycin.

The proportion of DRSP varies among demographic groups. The proportion of DRSP is highest among children younger than age two years (10). In 1994, a study in Atlanta found that Caucasians were more likely than Blacks to have invasive pneumococcal infections caused by drug-resistant pneumo-

\section{REFERENCES}

1. Prevention of pneumococcal disease: recommendations of the Advisory Committee on Immunization Practices (ACIP). Morb Mortal Wkly Rep 1997;46(RR-8):1-24.

2. Defining the public health impact of drug-resistant Streptococcus pneumoniae: report of a working group. Morb Mortal Wkly Rep 1996;45(RR-1 Suppl):1-20.

3. National Committee for Clinical Laboratory Standards. Performance standards for antimicrobial susceptibility testing \{NCCLS document no M100-S5]. Villanova: NCCLS, 1994.

4. National Committee for Clinical Laboratory Standards. Performance standards for antimicrobial susceptibility testing (8th informational supplement) [NCCLS document M100-S8]. Villanova: NCCLS, 1998.

5. Cetron MS, Breiman RF, Jorgensen JH, et al. Geographic variation of drug-resistant Streptococcus pneumoniae: population-based surveillance from nine locations. 97th General Meeting, American Society for Microbiology. Miami Beach, May 4 to 8 , 1997. (Abst C-371)

6. Assessment of national reporting of drug-resistant Streptococcus pneumoniae - United States, 1995-1996. Morb Mortal Wkly Rep 1996;45:947-9. cocci. Among Caucasian children age five years and younger, $41 \%$ of sterile site pneumococcal isolates were resistant to penicillin (12).

The geographic distribution of DRSP varies widely throughout the United States. Surveillance sites in the southeastern region of the United States report a higher prevalence of DRSP than other areas. From July 1995 to June 1996, Georgia and Tennessee reported that $15.1 \%$ and $19.3 \%$ of isolates, respectively, were resistant to penicillin (5). During that same time period, Toronto and San Francisco reported only 1.7\% and $1.9 \%$ penicillin resistance, respectively.

Five pneumococcal serotypes (6B, 9V, 14, 19A, and 23F) account for approximately half of disease caused by DRSP (7). Approximately $90 \%$ of invasive infections caused by DRSP are due to serotypes included in the 23 -valent pneumococcal vaccine.

\section{CONCLUSIONS}

Surveillance data in the United States have highlighted the public health burden attributable to pneumococcal disease, especially among the very young, the elderly and certain minority groups. In addition, surveillance has helped to identify a growing problem with drug resistance. Data about the prevalence of pneumococcal serotypes suggest, however, that much of the invasive disease among adults could be prevented through more widespread use of the 23-valent pneumococcal polysaccharide vaccine. The vaccine also would be effective for nearly all invasive disease in the elderly caused by drugresistant pneumococci. Several new vaccines are currently in development, including conjugate vaccines similar in structure to those used against Haemophilus influenzae type b. Expanded surveillance for pneumococcal disease will be needed to measure the impact of new vaccines and to assess efforts to slow emerging drug resistance.

7. Butler JC, Hoffman J, Cetron MS, et al. The continued emergence of drug-resistant Streptococcus pneumoniae in the United States: an update from the Centers for Disease Control and Prevention's Pneumococcal Sentinel Surveillance System. J Infect Dis 1996;174:986-93.

8. Schuchat AS, Robinson K, Wenger JD, et al. Bacterial meningitis in the United States in 1995. N Engl J Med 1997;337:970-6.

9. Spika JS, Facklam RR, Plikaytis BD, Oxtoby MJ. Pneumococcal Surveillance Working Group. Antimicrobial resistance of Streptococcus pneumoniae in the United States, 1979-1987. J Infect Dis 1991;163:1273-8.

10. Breiman RR, Butler JC, Tenover FC, Elliott JA, Facklam RR Emergence of drug-resistant pneumococcal infections in the United States. JAMA 1994;271:1831-5.

11. Cetron MS, Breiman RF, Jorgensen JH, et al. Multi-site population-based surveillance for drug-resistant Streptococcus pneumoniae. 97th General Meeting, American Society for Microbiology. Miami Beach, May 4 to 8, 1997. (Abst C-283)

12. Hofmann J, Cetron MS, Farley MM, et al. The prevalence of drug-resistant Streptococcus pneumoniae in Atlanta. N Engl J Med 1995;333:481-6. 


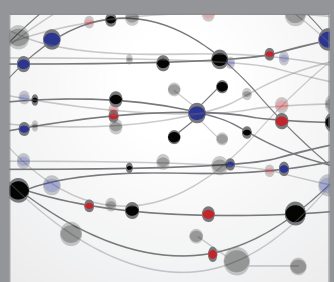

The Scientific World Journal
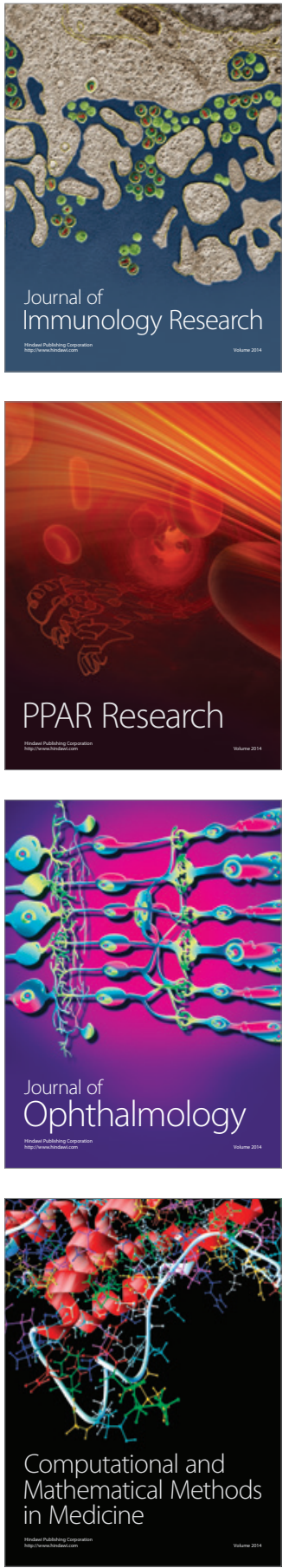

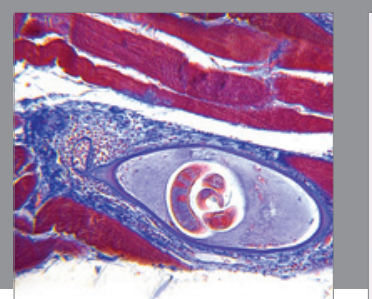

Gastroenterology Research and Practice

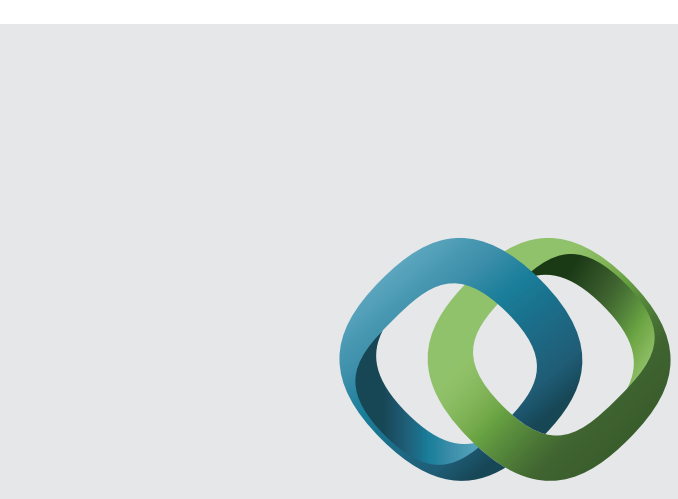

\section{Hindawi}

Submit your manuscripts at

http://www.hindawi.com
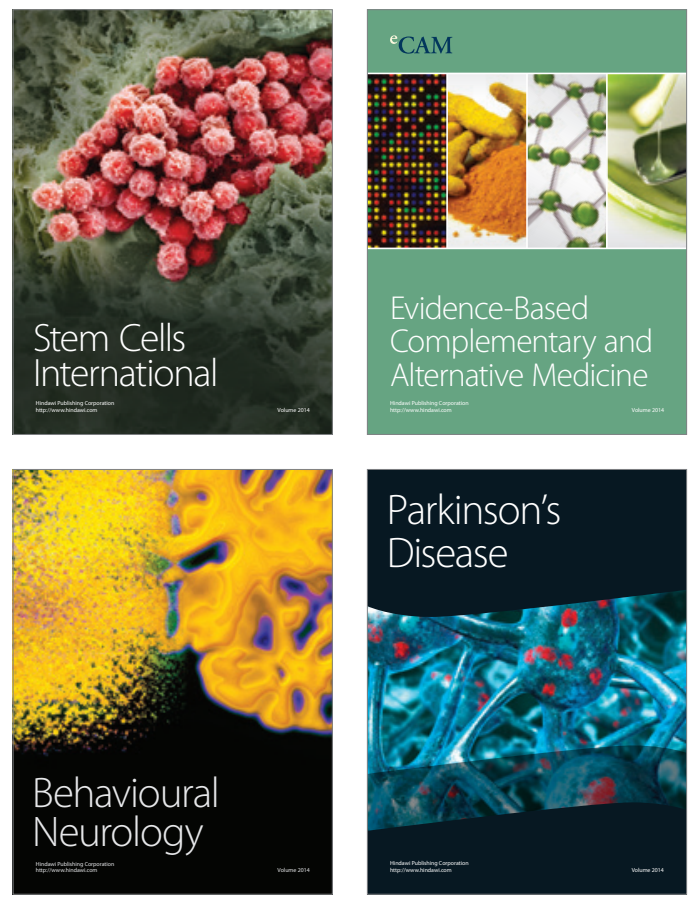
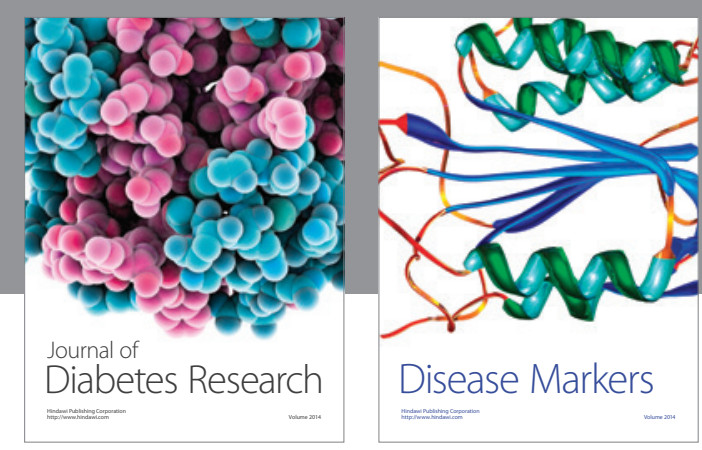

Disease Markers
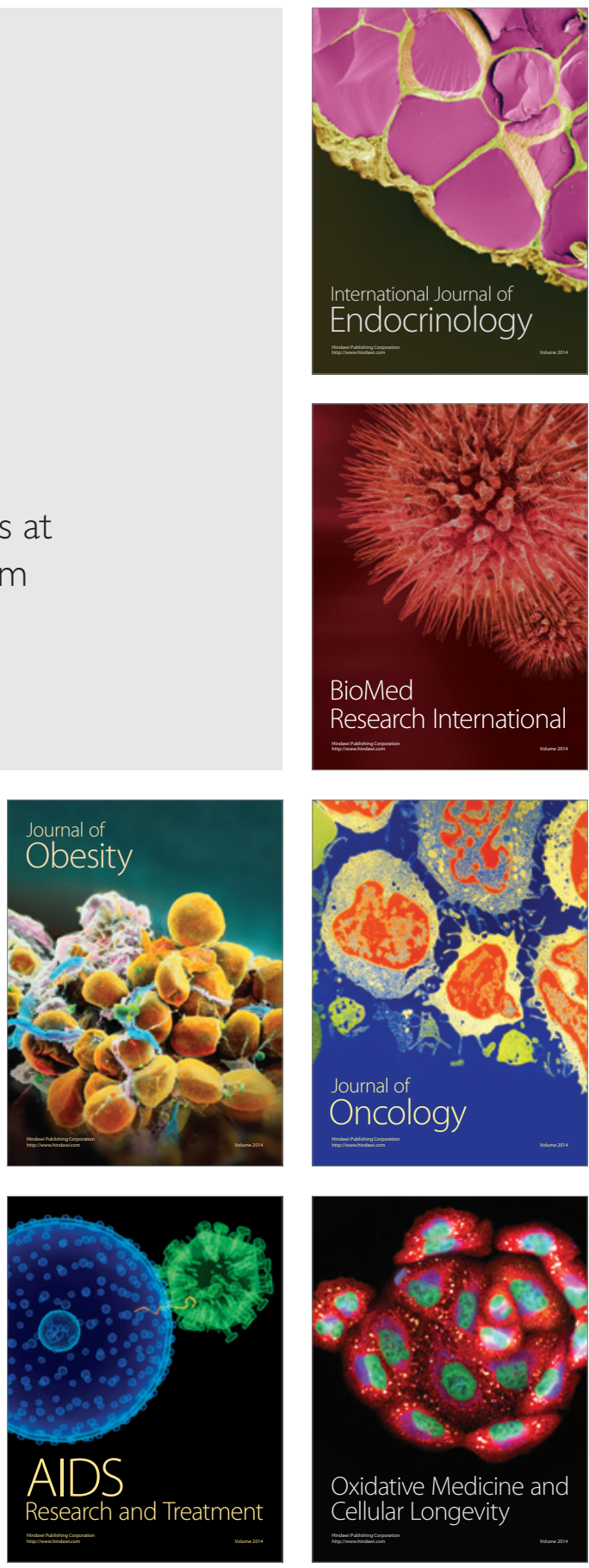